\title{
Measurements of Complex Harmonic Current on Two Wind turbines
}

\author{
K. Yang ${ }^{1}$ and M. H. J. Bollen ${ }^{1,2}$ \\ ${ }^{1}$ Electric Power Engineering Group \\ Luleå University of Technology \\ 93187 Skellefteå (Sweden) \\ kai.yang@ltu.se \\ ${ }^{2}$ STRI AB \\ 77180 Ludvika, Sweden \\ math.bollen@stri.se
}

\begin{abstract}
The paper presents measurements of complex harmonic current of two modern wind turbines. The complex current has been obtained with a $5 \mathrm{~Hz}$ resolution up to $2.8 \mathrm{kHz}$. The distribution of complex current has been studied in the complex plane. Distinction has been made between low-order integer harmonics, interharmonics and high-order harmonics: low-order integer harmonics are distributed around a center offset from the origin of the complex plane; interharmonics and high-order harmonics are distributed around the origin with a uniformly distributed phase-angle.
\end{abstract}

\section{Key words}

Renewable electricity production, Wind power generation, Power quality, Harmonic analysis.

\section{Introduction}

Wind turbines equipped with power electronics emitting harmonic distortion has been studied in a lot of publications $[1,2,3]$.

Theoretical study has been performed on the harmonic current in the complex plane in a number of publications $[4,5]$. The complex harmonics show a certain distribution in the complex plane.

Study based on measurements has been performed on lowfrequency and a high-frequency harmonics, in which it is concluded that low frequency phase-angle presents a narrow normal-type distribution and high frequency phaseangle a uniform distribution [6].

This paper will study the distribution of the complex current (with $5 \mathrm{~Hz}$ resolution in frequency) up to $2.8 \mathrm{kHz}$. The characteristics of each harmonic and interharmonic will be studied according to measurements of two individual wind turbines. The distribution of the complex current has been assessed in a quantitative way.
Section 2 presents the setup of the measurements and information about the measurement objective; Section 3 presents the measurements with the complex current, as well as the quantification of phase-angle distribution; and the conclusion is summarized in Section 4.

\section{Measurement from Two Wind Turbines}

\section{A. Measurement Objective}

The two measured individual wind turbines, which are from different manufacturers, have been measured at the medium voltage (MV) side of the turbine transformers:

- Turbine A: it is a type-3 wind turbine with a rated-power $2.5 \mathrm{MW}$. It is equipped with a sixpole doubly-fed asynchronous generator (DFIG). A partial rated converter with IGBT technology is applied in the wind turbine. The turbine transformer is placed beside the turbine tower. The measurement point (MV) is with a rated voltage $22 \mathrm{kV}$ and a rated current $66 \mathrm{~A}$.

- Turbine B: this is a type-4 wind turbine, with a rated power of $2 \mathrm{MW}$. The full-power converter is with IGBT technology, which fed the power into the grid through a turbine transformer. The measurement point (MV) is with a rated voltage $10 \mathrm{kV}$ and a rated current $116 \mathrm{~A}$.

\section{B. Measurement Approach}

The three-phase waveforms have been recorded by the instrument Dranetz PX5 using the conventional voltage and current transformers, with a sufficient accuracy for the frequency range up to a few $\mathrm{kHz}[7,8]$.

The waveforms were captured with 200-ms windows with a sampling frequency of $12.8 \mathrm{kHz}$. The Discrete Fourier Transform (DFT) has been applied using a rectangular window of 10 -cycles. It results in a spectrum 
with a $5 \mathrm{~Hz}$ frequency resolution. An extra test has been done with a rectangular window of 8 cycles, to exclude the error by the DFT. The results are the same as those from using the 10-cycle waveform.

\section{Complex Harmonic current}

A. Low-order Complex Harmonic
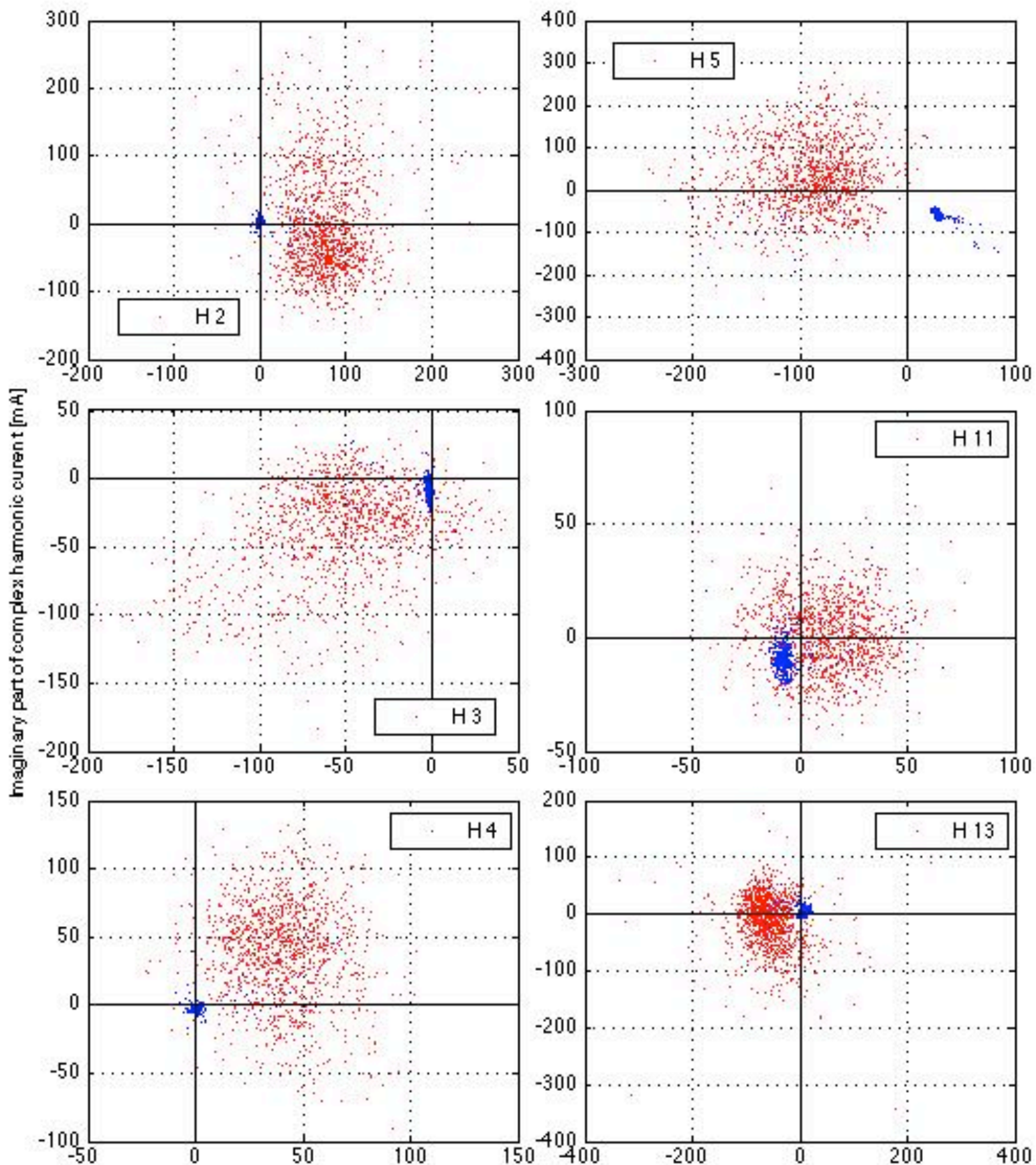

Real part of complex hamonic curent [mA]

Fig. 1. Plots of complex current of Turbine A at low order harmonics. 
The blue dots represent the complex current when the wind turbine is idle and supplied by the grid; while the red dots represent the complex emission when the wind turbine is generating power into the grid.

The complex harmonics (at low order integer harmonics) shown in the above figure are distributed around a center point, which is not the origin of the complex plane.
Harmonics are different from order to order, by the shape of the distribution.

Turbine $\mathrm{B}$ has been measured with a duration of 13 days, where 1864 scatter points (complex current obtained from waveforms) are recorded in the complex plane. The complex currents are plotted as in Fig. 2.
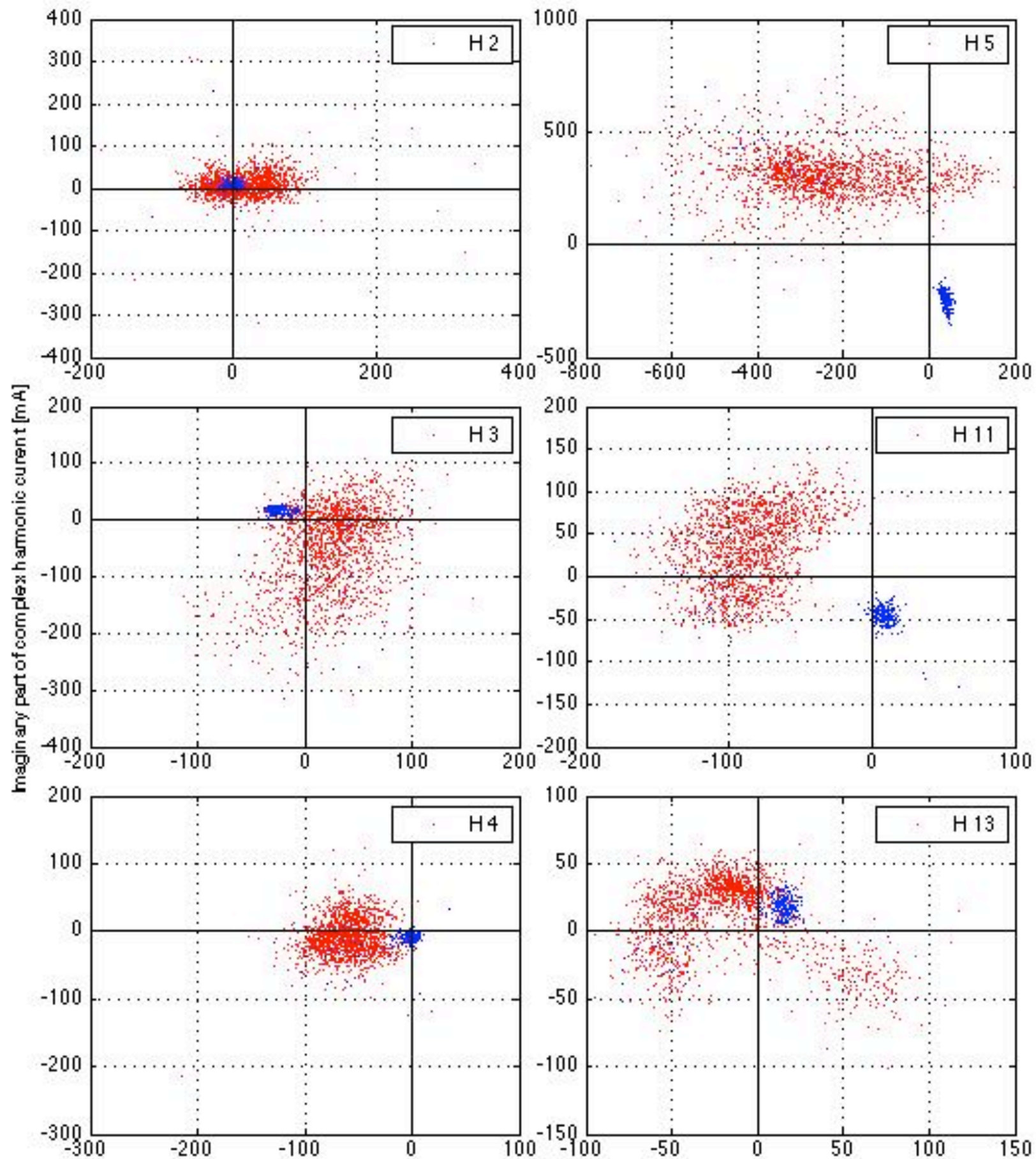

Real part of complex hamonic curent [mA]

Fig. 2. Complex harmonic current of Turbine B at lower orders. 
The above figure holds the same trend as Turbine A that, low-order integer harmonics are distributed around a center point which is not the origin of the complex plane.

B. High-order Complex Harmonics and interharmonics
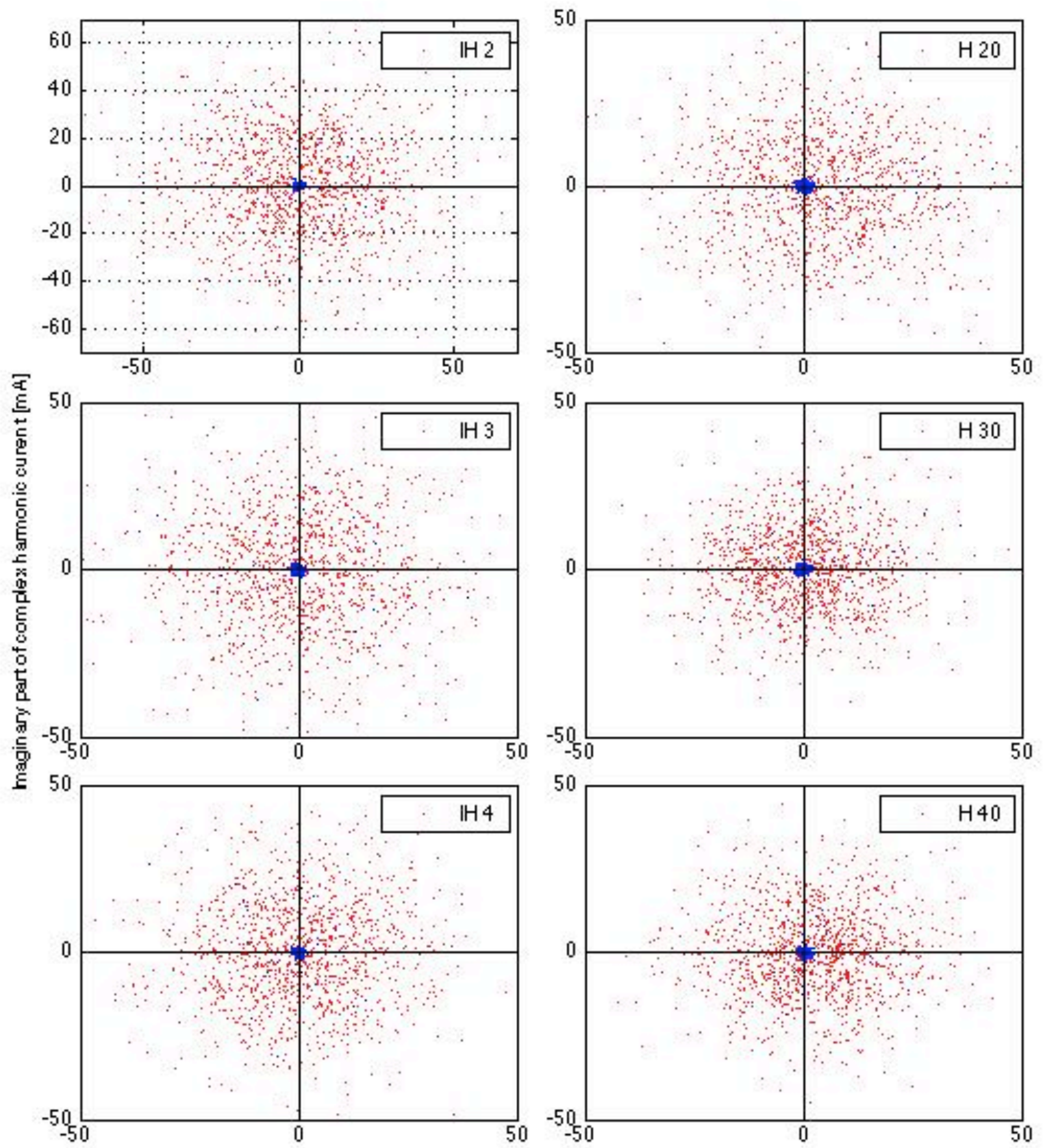

Real part of complex hamonic curent [mA]

Fig. 3. Complex current of interharmonics and high-order harmonics of Turbine A. 

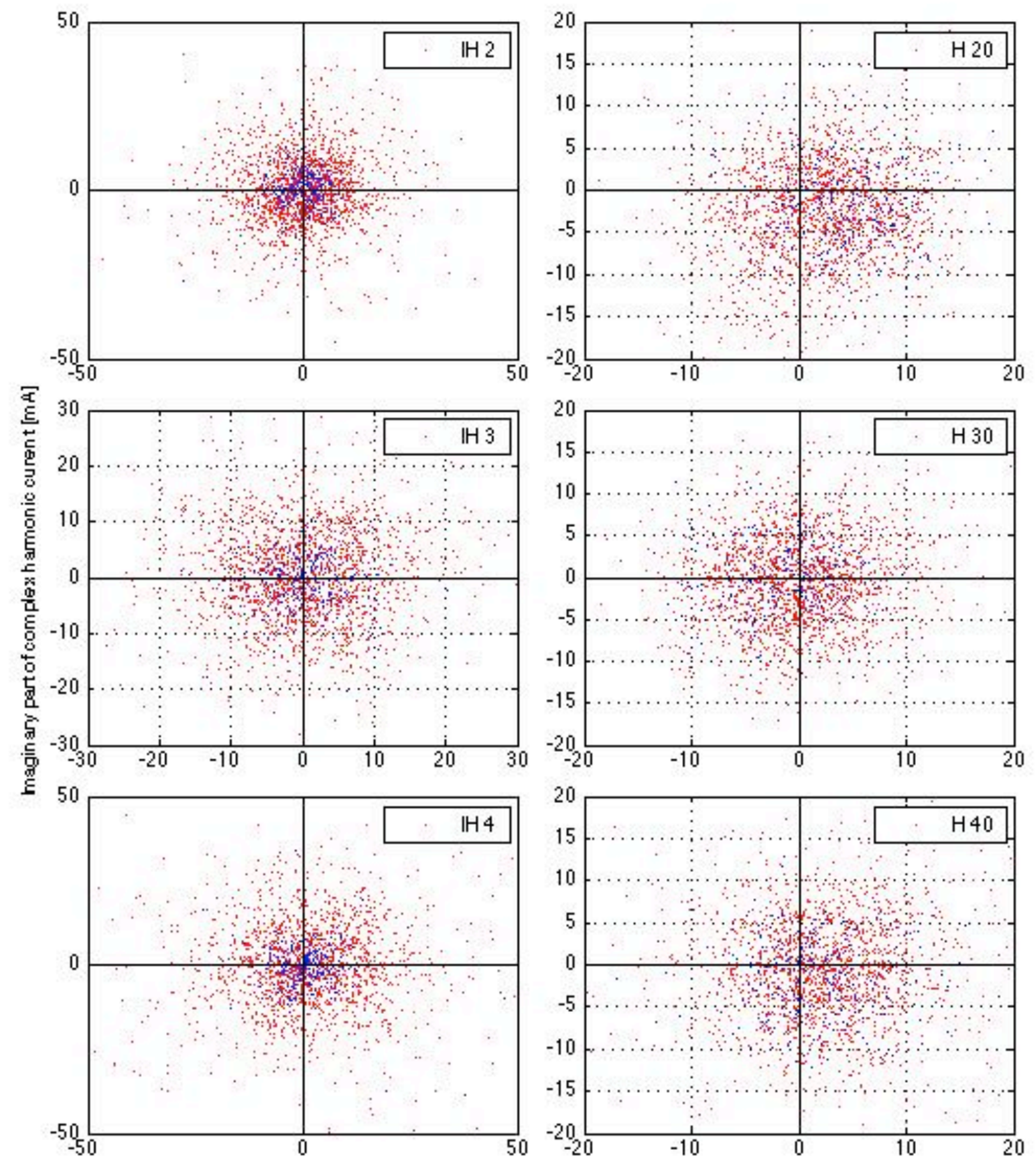

Real part of complex hamonic curent [mA]

Fig. 4. Complex current of interharmonics and high-order harmonics of Turbine B

Both for Turbine A and Turbine B, interharmonics and high-order harmonics are distributed around the origin of the complex plane. And the scatter points are randomly distributed in the complex plane. It indicates that the phase-angles of the interharmonics and high-order harmonics are uniformly distributed.

\section{Distribution of the phase angle}

A chi-square goodness-of-fit test has been used to test the distribution of the phase-angles of the complex current, up to the frequency $2.8 \mathrm{kHz}$. The test is based on the Matlab 
function chi2gof, with a 95 percent significance level. Only the complex currents at the positive power production have been chosen in the test. The results of the distribution type test for Turbine $\mathrm{A}$ and Turbine $\mathrm{B}$ are shown in Fig. 5 and Fig. 6, respectively.
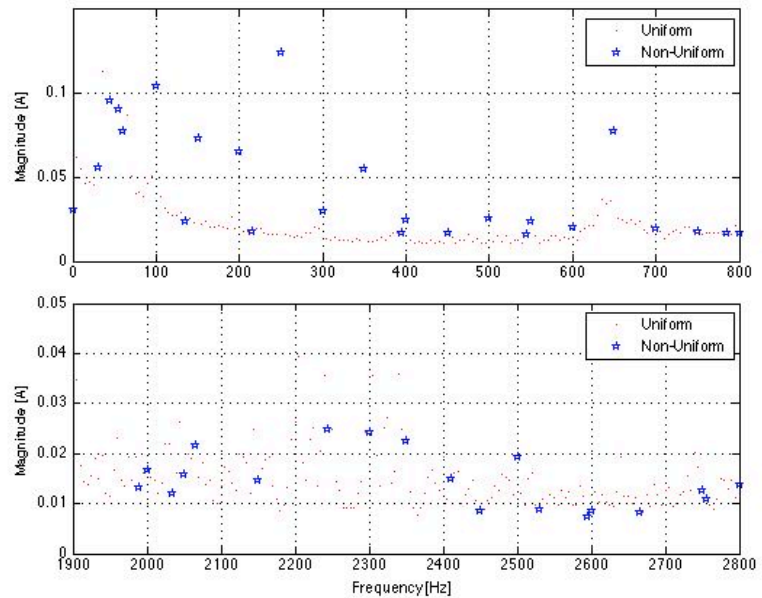

Fig. 5. Distribution type (phase-angle) of Turbine A.
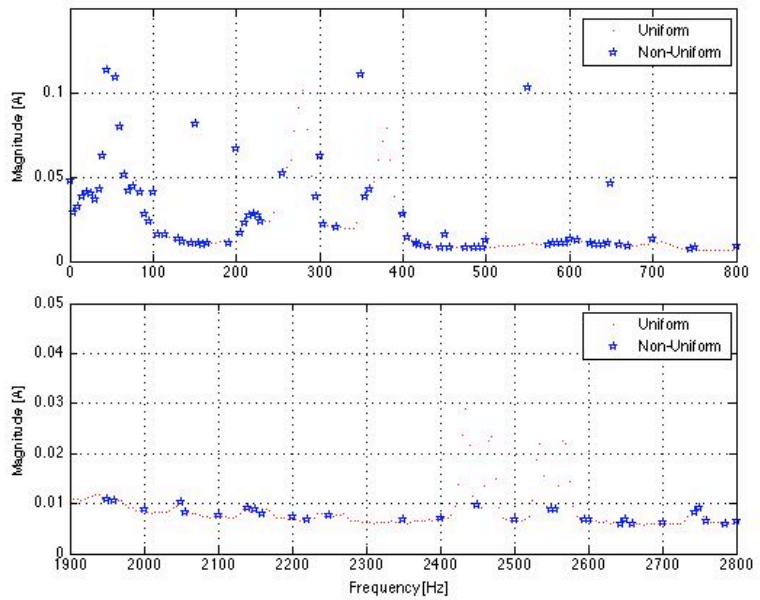

Fig. 6. Distribution type (phase-angle) of Turbine B.

The red dots represent the uniformly distribution of the phase-angle at the certain frequency. Whereas the blue stars represent the non-uniform distribution for the phaseangles.

The red dots represent frequency components for which the chi-square test indicated at least $95 \%$ confidence for a uniform distribution of the phase angle. The blue starts indicate frequencies where such a high confidence could not be reached. This may be due to lack of measurement points or because the distribution simply is not uniform. For simplicity, those will be referred to as "non-uniform distribution".

The common observations from the both two turbines are that: the non-uniform distribution is mainly at the low frequencies, especially for the integer harmonics; the uniform distribution is mainly at the interharmonics and high-order harmonics.

\section{Conclusion}

This paper presents measurements of complex harmonic current from two types of wind turbines. FFT has been applied to obtain spectra with $5-\mathrm{Hz}$ resolution during a period of a few weeks.

The scatter plots of the complex harmonic current at integer harmonics and interharmonics have been presented. Characteristics are different for harmonics and interharmonics, low orders and high orders. Low-order harmonics present a non-uniform distribution of the phase angle; while interharmonics and high-order harmonics present a uniform distribution. At the uniform distribution orders, phase-angles of the frequency component are randomly distributed in the complex plane.

With the non-uniform and uniform distributions of different frequency components, the representation of the distortion will be impacted in a long-term assessment of wind turbine distortion and distortion with in a wind park with a number of wind turbines. The harmonic/interharmonic components with a uniform phase-angle will cancel each other more than these with non-uniform distributed phase-angles.

Further study is needed to quantify the effect of the cancellation at each harmonic order, and the final harmonic/interharmonic level in a wind park as a whole.

\section{Acknowledgement}

The authors would like to thank the financial support by the Vindforsk program and by Skellefteå Kraft Elnät AB. Many thanks to Mats Wahlberg, with Skellefteå Kraft Elnät $\mathrm{AB}$, for the measurements.

\section{References}

[1] T. Ackermann and etc., Wind Power in Power Systems, $1^{\text {st }}$ ed. John Wiley \& Sons, Ltd, 2005.

[2] M. Bollen and F. Hassan, Integration of Distributed Generation in the Power System, $1^{\text {st }}$ ed. Wiley-IEEE Press, 2011.

[3] R. Dugan, M. McGranaghan, S. Santosa, and H. Beaty, Electric Power Systems Quality, second Edition. Now York: McGraw-Hill, 2003.

[4] E. Kazibwe, T. Ortmeyer, and M. Hammam, "Summation of probabilistic harmonic verctors [Power System]", IEEE Transactions on Power Delivery, vol.4, pp.621-628, Jan 1989.

[5] A. Cavallini, R. Langella, A. Testa, and F. Ruggiero, "Gaussian modelling of harmonic verctors in power system", in Proceedings $8^{\text {th }}$ International Conference on Harmonics and Quality of Power Proceedings, 1998, vol.2, oct, 1998.

[6] S. T. Tentzerakis and S. A. Papathanassiou, "An investigation of the harmonic emissions of wind turbines," IEEE Transactions on Energy Conversion, vol. 22, no. 1, pp. 150 158, March 2007.

[7] J. Arrillaga, N. Watson, S. Chen, Power system quality assessment, 1st Edition, John Wiley \& Sons, 1999.

[8] J. Meyer, P. Schegner, K. Heidenreich, Harmonic summation effects of modern lamp technologies and small electronic household equipment, in: Proceedings of The 21st International Conference and Exhibition on Electricity Distribution, Frankfurt, Germany, 2011. 